\title{
Apolipoprotein B is a new target of the GDNF/RET and ET-3/EDNRB signalling pathways
}

\author{
C. EVANGELISTI, ${ }^{\star}$ F. BIANCO,${ }^{\star}$ L. M. PRADELla, ${ }^{\star}$ A. PUliti $, \dagger,+$ A. GOLdONI, ${ }^{\star}$ I. SBRANA, $\S$ M. ROSSI, $\S$ \\ M. VARGIOlU, ${ }^{\star}$ M. SERI, ${ }^{\star}$ G. ROMEO ${ }^{\star}{ }^{\star}$ V. STANGHellini, $\uparrow$ R. DE GIORGIO $\uparrow$ \& E. BONORA ${ }^{\star}$ \\ ${ }^{\star}$ Medical Genetics Unit, St.Orsola-Malpighi Hospital, University of Bologna, Bologna, Italy \\ $\dagger$ Department of Pediatric Sciences 'Giovanni De Toni', University of Genova, Genova, Italy \\ $\$$ Molecular Genetics and Cytogenetics Unit, Gaslini Institute, Genova, Italy \\ $\S$ Department of Biology, University of Pisa, Pisa, Italy \\ -Department of Clinical Medicine, and Digestive Diseases and Internal Medicine, St.Orsola-Malpighi Hospital, University \\ of Bologna, Bologna, Italy
}

\begin{abstract}
Background GDNF/RET and Endothelin-3 (ET-3)/ EDNRB regulate survival, differentiation, migration, and proliferation of neural crest-derived cells. Although several RET and EDNRB signalling mediators have been characterized, most of the genes targeted by these two pathways are still largely unknown. We focused our study on apolipoprotein $B$ (APOB) as a novel target gene of the RET and EDNRB pathways, based on previous data obtained using a Caenorhabditis elegans strain mutant for the homologue of mammalian ECE1. Methods Molecular and cellular studies of Apob were performed in the murine Neuro2a cells, an in vitro model for studying neural crest-derived cell development, along with a mouse knock-in for the Hirschsprung-associated mutation Ret ${ }^{\mathrm{C} 620 \mathrm{R}}$. Silencing for Apob and Ret has been performed via shRNA. Key Results GDNF/RET and ET-3/EDNRB cooperated in inducing neuronal differentiation resulting in Apob activation in Neuro2a cell line. Apob expression was downregulated in mouse embryos homozygous for the Ret ${ }^{\mathrm{C} 20 \mathrm{R}}$ mutation and presenting a severe Hirschsprung phenotype. Ret silencing prevented Apob expression increase. MAPK P38 kinase activation evoked Apob expression via GDNF/RET signalling in Neuro2a cells.
\end{abstract}

Address for Correspondence

Roberto De Giorgio, MD, PhD, AGAF, Department of Clinical Medicine, Bldg \#5, St.Orsola-Malpighi Hospital, Via Massarenti 9, 40138, University of Bologna, Bologna, Italy.

Tel: +39 051636 3558; fax: +39 0513458 64;

e-mail: roberto.degiorgio@unibo.it

Received: 20 January 2012

Accepted for publication: 9 July 2012
A p53-dependent repressor element in Apob promoter resulted in a reduced Apob expression. Silencing of Apob reduced HuD protein expression. Conclusions et Inferences $A p o b$ is a novel downstream target of the RET/EDNRB pathways with a role in neuronal survival and maintenance, as indicated by its effect on $\mathrm{HuD}$ expression. Our data provide a conceptual framework to investigate and establish the role of $A P O B$ gene in severe gut dysmotility.

Keywords apolipoprotein B, enteric nervous system, Hirschsprung's disease, Neuro2a cell line, Ret ${ }^{\text {C620R }}$.

\section{INTRODUCTION}

An intact and functioning enteric nervous system (ENS) is required for life. The segmental absence of the ENS, which occurs either as a congenital [Hirschsprung's disease (HSCR)] or acquired defect, ${ }^{1}$ causes a severe impairment of gastrointestinal transit. Hirschsprung's disease is characterized by the absence of enteric ganglia in the intestinal tract, with an incidence of approximately $1 / 5000$ births affecting more men than women $\left(4: 1\right.$ ratio)., ${ }^{2,3}$

$R E T$ is the main gene involved in HSCR: the majority of patients harbor either heterozygous mutations in the coding region or a heterozygous or homozygous hypomorphic allele in a conserved enhancer sequence in intron 1., ${ }^{4,5}$ Mutations in other genes encoding proteins involved in either RET or Endothelin receptor type-B (EDNRB) signalling pathways are found in a small percentage of HSCR patients. ${ }^{2,3}$

RET codes for a tyrosine kinase transmembrane receptor, with two alternative isoforms at the 
C-terminal recruiting different signalling factors. ${ }^{6}$ Activation of RET occurs upon self-dimerization, induced by the binding of glial cell line-derived neurotrophic factor (GDNF) family ligand to glycosylphosphatidylinositol-anchored co-receptor GDNF family receptor $\alpha$ and resulting in the activation of three main kinases, Akt, ERK, and MAPK P38. ${ }^{4}$ RET activation regulates ENS normal development by modulating survival, differentiation, migration, and proliferation of enteric neural crest-derived cells.

EDNRB is a G-coupled receptor that binds any of three endothelins (ET-1, -2, -3), ${ }^{7}$ produced as preproendothelins and proteolytically activated by Endothelin-converting Enzyme-1 (ECE-1). The crosstalk between GDNF/RET and ET-3/EDNRB pathways in coordinating ENS development has been demonstrated in in vitro and in vivo studies. ${ }^{8-11}$ Nevertheless, the molecular bases of this crosstalk remain to be elucidated.

This study was designed to investigate apolipoprotein $\mathrm{B}$ (APOB) as a novel target of the crossregulation between RET and EDNRB pathways, as emerged from previous studies using the Caenorhabditis elegans strains mutant for the mammalian ECE1 homologue. ${ }^{12}$ $A P O B$ is expressed in two isoforms, APOB100 and APOB48, the former being synthesized in the liver as an essential component of lipoproteins, and the latter being generated by RNA editing in the gut for chylomicron production. ${ }^{13} A P O B$ mutations cause familial hypobetalipoproteinemia ${ }^{14}$ and familial defective apolipoprotein B-100. ${ }^{15}$

A correlation between APOB and the genes involved in ENS development, such as EDNRB and RET, has not been reported so far. However, Apob knockout mice showed severe neural tube defects suggesting a role for APOB during embryogenesis and in particular with regard to neural crest development. ${ }^{16}$ We therefore focused on the Neuro2a murine cell line, an in vitro model for studying neural crest development and neuronal differentiation, ${ }^{17,18}$ along with a mouse knock-in for the mutation Ret $^{\text {C620R }}$ associated to HSCR $^{19}$ to investigate the role of Ret and Ednrb signalling pathways in Apob expression and to identify the molecular partners involved in this process. We demonstrated that $A p o b$ expression is actually activated by GDNF/Ret via MAPK P38 and modulated by ET-3/Ednrb. Furthermore, we identified p53 as a transcription factor involved in the regulation of Apob expression. Taken together our data provide a basis to investigate the role of $A P O B$ in the clinical setting specifically focusing on severe gut dysmotility disorders.

\section{MATERIALS AND METHODS}

\section{Cell cultures and growth factor treatments}

Murine Neuro2a cells were grown in DMEM, supplemented with $10 \%$ fetal bovine serum (FBS), $2 \mathrm{~mm}$ L-glutamine, at $37{ }^{\circ} \mathrm{C}$ in a $5 \%$ $\mathrm{CO}_{2}$ humidified atmosphere. SK-N-MC and SK-N-BE were grown in DMEM/F12 1/1 v/v and RPMI, respectively, supplemented with $2 \mathrm{~mm}$ L-glutamine, $100 \mathrm{U} \mathrm{mL}^{-1}$ penicillin, $100 \mu \mathrm{g} \mathrm{mL}^{-1}$ streptomycin and $10 \%$ FBS at $37^{\circ} \mathrm{C}$ in a $5 \% \mathrm{CO}_{2}$ humidified atmosphere.

After $16 \mathrm{~h}$ serum starvation, Neuro2a cells were treated with $50 \mathrm{ng} \mathrm{mL}^{-1}$ GDNF (R\&D Systems, Minneapolis, MN, USA) and $300 \mathrm{nmol} \mathrm{L}^{-1}$ ET-3 (Sigma-Aldrich, St. Louis, MO, USA), separated and in combination for different times. To test RETactivated signalling pathways, Neuro2a cells were shifted to a serum free medium in the presence of kinase inhibitors before GDNF treatment, as follows: TCN (Triciribine V) (Akt inhibitor) $10 \mu \mathrm{m} 24 \mathrm{~h}$; SB202190 (MAPK38 inhibitor) $20 \mu \mathrm{m} 24$ h; PD98059 (ERK inhibitor) $40 \mu \mathrm{m} 24 \mathrm{~h}$. To test Neuro2a cell growth and differentiation upon GDNF and/or ET-3 treatment, Neuro2a cells were seeded on PolyD lysine (Sigma-Aldrich) precoated 24-well plates to a final density of 2500 or 5000 cells. The following day the medium was replaced with DMEM supplemented with $2 \mathrm{~mm}$ L-glutamine, $100 \mathrm{U} \mathrm{mL}^{-1}$ penicillin, $100 \mu \mathrm{g} \mathrm{mL}^{-1}$ streptomycin, $0.5 \mu \mathrm{g} \mathrm{mL}^{-1}$ DMPH4, $2.5 \mu \mathrm{g} \mathrm{mL}^{-1} \mathrm{~L}-\mathrm{GSH}, 50 \mu \mathrm{g} \mathrm{mL}^{-1}$ L-ascorbic acid, and N2 Supplement (synthetic medium) and cells were treated with GDNF $\left(10 \mathrm{ng} \mathrm{mL}^{-1}\right)$ and ET-3 $\left(300 \mathrm{nmol} \mathrm{L}{ }^{-1}\right)$ separately and in combination. Triplicate wells were studied for each condition, including three wells in which cells were grown in the synthetic medium without the addition of any growth factors (control cultures). For time course observations, cells were plated in multiple 24-wells plates, fixed in methanol $\left(-20^{\circ} \mathrm{C}\right)$ at a particular time point (every $24 \mathrm{~h}$ ) for up to 5 days. Half of the medium was replaced once every 2 days. At the end of the treatment, cell growth was evaluated by counting cells present in each well using phase-contrast microscopy with the help of a grid placed in the ocular and lines drawn on a transparent film attached to the 24-multiwell plate in such a way as to obtain distinct fields to be evaluated separately. Cell differentiation was evaluated by a detailed analysis of cell morphology by phasecontrast microscopy. Cells were classified according to the presence and shape of neurites in six groups: undifferentiated, round-shaped cells; cells with one neurite (monopolar), cells with two opposite neurites (bipolar); cells with two opposite and branched neurites (branched-bipolar), cells with many neurites (star cells); cells with one prevalent branched neurite (branched) (Figure S1).

\section{Mice knock-in $\operatorname{Ret}^{\mathrm{C620R}}$}

Ret ${ }^{\text {C620R }}$ mice were grown at Charles River animal facility (Lecco, Italy). Experimental procedures were approved by the ethical committee for animal experimentation of the University of Bologna, in accordance with European legislation (European Communities Council directive of November 24, 1986, 86/609/EEC).

\section{Western blotting}

Whole-cell lysates were obtained by scraping cells directly in IP Buffer 1X containing deoxycholate (Sigma-Aldrich) and a cocktail of protease inhibitors (Roche Diagnostics, Indianapolis, IN, USA) and phosphatase inhibitors (Sigma-Aldrich) on ice for $15 \mathrm{~min}$. 
Cells were collected and lysed through an insulin syringe. Protein concentration was evaluated with the DC protein concentration assay Kit (Biorad, Hercules, CA, USA). Proteins were denatured by heating at $95{ }^{\circ} \mathrm{C}$ for $5 \mathrm{~min}$ in Laemli buffer, separated by SDS gel electrophoresis, transferred onto nitrocellulose membrane (GE Healthcare, Chalfont St. Giles, UK), and subjected to Western blotting. Bands were visualized using the ECL method (GE Healthcare).

Primary antibodies used were as follows: anti-PAN-RET $1: 200$, anti-phospho-Akt $1: 1000$, anti-total Akt $1: 1000$, anti-phosphoErk 1: 1.000, anti-total Erk 1: 1000 (Santa Cruz, Biotechnology Inc., Santa Cruz, CA, USA), anti-phospho MAPK P38 kinase $1: 1000$, anti-MAPK P38 kinase 1: 1000 (Cell Signaling, Danvers, MA, USA), anti-HuD $1: 100$ (Invitrogen, Carlsbad, CA, USA), anti$\gamma$-tubulin $1: 10$ 000, anti-vinculin 1:30000 (Sigma-Aldrich).

\section{Ret and Apob silencing via shRNA}

Neuro2a cells $\left(5 \times 10^{5}\right)$ were transfected either with the pRSshRNA vectors (OriGene, Rockville, MD, USA) (specific for mouse Ret, TR1504590, and Apob silencing, TI562711), or the empty vector, or the scramble vector (control) using lipofectamine (Invitrogen). After $24 \mathrm{~h}$, cells were divided into the number of wells necessary for GDNF stimulation. The next day, cells were shifted to a serum-free medium for $16 \mathrm{~h}$. GDNF treatment and protein extraction were performed as previously described. Lack of Ret expression was verified via Western blotting using the PANRET antibody $(1: 200)$. RNA was extracted and Apob silencing was verified by real-time quantitative RT-PCR as described below.

\section{Immunocytochemisty}

Neuro2a cells were fixed in $4 \%$ PFA, washed in PBS and incubated in a $0.3 \% \mathrm{H}_{2} \mathrm{O}_{2} /$ Methanol solution for $15 \mathrm{~min}$, then incubated for 60 min at $4{ }^{\circ} \mathrm{C}$ in blocking solution ( $1 \%$ BSA/PBS) and washed three times for $30 \mathrm{~min}$ in PBS. Two primary anti-RET antibodies were used obtaining similar results: anti-Ret antibody (R\&D Systems) and anti-PAN-RET (Santa Cruz). Cells were incubated with primary antibody at a $1: 200$ dilution for $2 \mathrm{~h}$, washed in PBS and incubated for $1 \mathrm{~h}$ with biotinylated secondary antibody (Santa Cruz) at a $1: 200$ dilution. Slides were washed three times for $30 \mathrm{~min}$ in PBS. Staining was performed using the Vectastain Elite ABC kit (Vector, Burlingame, CA, USA) and the DAB substrate kit for peroxidase (Vector). SK-N-MC and SK-N-BE neuroblastoma cell lines were used as negative and positive control, respectively. ${ }^{20,21}$

\section{Immunohistochemistry}

Immunohistochemistry was performed on paraffin-embedded adult human colon tissues (patients routinely operated for uncomplicated colon cancers commonly used as controls for immunohistochemical experiments) according to protocols validated in our laboratory. ${ }^{22}$ Briefly, tissue sections were treated to remove paraffin embedding by three sequential washes in xylene and graded ethanol. Antigen unmasking was performed by heating sections at $95{ }^{\circ} \mathrm{C}$ in $10 \mathrm{~mm}$ Sodium citrate buffer, $\mathrm{pH}$ 6.0, for $10 \mathrm{~min}$ and subsequent cooling at room temperature for $30 \mathrm{~min}$. To reduce endogenous peroxidases, tissue sections were treated with an ad hoc blocking kit (GeneTex Inc., Irvine, CA, USA) and then incubated for $16 \mathrm{~h}$ at $4{ }^{\circ} \mathrm{C}$ with primary antibodies, i.e. antihuman APOB diluted 1:100 (ABCAM, Cambridge, UK); antihuman NSE diluted 1:250 (Santa Cruz). Fluorescent secondary antibodies were diluted in Triton-X $(1: 300)$ and incubated for $60 \mathrm{~min}$ at room temperature, before subsequent dehydration and visualization under a LEICA DMLB fluorescent microscope.

\section{Quantitative real-time polymerase chain reaction (qRT-PCR) analysis}

Total RNA was extracted using the Trizol method and was reverse transcribed using Superscript III kit and random hexamers (Invitrogen). Primers for Apob, Actb were designed with Primer3 (http://www.genome.wi.mit.edu/cgi-bin/primer/primer3_www.cgi) and RTPrimerDB (http://medgen.ugent.be/rtprimerdb/). Quantitative real-time polymerase chain reaction was performed with SYBRGreen, $0.5 \mu \mathrm{m}$ primers, in an ABI 7500 Real-Time PCR System (Applied Biosystems, Foster city, CA, USA). All target genes were normalized with the corresponding endogenous control using the $\Delta C_{t}$ comparative method; $A p o b$ expression levels were normalized to beta-actin $(A c t b)$.

Expression of Ret, Gfra1 and Ednrb in Neuro2a cells was assessed by RT-PCR using mouse brain RNA as positive control. All primers used in the present work are available on request.

\section{Expression of Apob in mouse embryos $\operatorname{Ret}^{\mathrm{C620R}}$}

$\operatorname{Ret}^{\mathrm{C} 620 R} / \operatorname{Ret}^{\mathrm{C} 620 \mathrm{R}},+/$ Ret $^{\mathrm{C} 620 R}$ and wild-type embryos were removed by Caesarian section from pregnant carrier mothers at 16 days post coitum (d.p.c.) and genotyped as described previously. ${ }^{19}$ Tissues were preserved in RNAlater (QIAGEN, Valencia, CA, USA) and total RNA was extracted using the Trizol method. qRT-PCR was performed as described above.

\section{Mouse Apob promoter analysis}

Mouse $A p o b$ upstream region was amplified using primer $5^{\prime}$ GGGCTCGAGGGCTTATGGCCTCTGTATGC-3' inserting an XhoI restriction site at the $5^{\prime}$ end, and primer $5^{\prime}$ GGGAAGCTTCCTGCTGCTTGCTCTACAGG-3' inserting a HindIII restriction site at the $3^{\prime}$ at the following condition: $60 \mathrm{ng}$ mouse genomic DNA, $0.4 \mu \mathrm{m}$ primers, $0.4 \mathrm{~mm}$ dNTPs, $2.5 \mathrm{~mm} \mathrm{MgCl}_{2}$, DMSO 5\%, $0.2 \mu \mathrm{L}$ Platinum Taq in a final volume of $50 \mu \mathrm{L}$. The PCR amplification was performed as follows: $10 \mathrm{~min} 94^{\circ} \mathrm{C}, 1 \mathrm{~min}$ at $94{ }^{\circ} \mathrm{C}, 30 \mathrm{~s}$ at $62^{\circ} \mathrm{C}, 1 \mathrm{~min}$ at $72{ }^{\circ} \mathrm{C}, 46$ cycles. The PCR product was inserted in the pGL3 basic vector and different deleted clones were generated with the Erase-a-base kit (Promega, Madison, WI, USA). The mutated promoter construct at $-986 \mathrm{bp}$ was generated with the QuickChange sitedirected mutagenesis kit (Agilent, Santa Clara, CA, USA). $400 \mathrm{ng}$ of each construct was independently co-transfected with $400 \mathrm{ng}$ of the control vector containing the renilla luciferase reporter into $2 \times 10^{5}$ Neuro2a cells using lipofectamine in duplicate. $24 \mathrm{~h}$ after transfection, cells were shifted to a serum free medium for $16 \mathrm{~h}$, treated for $1 \mathrm{~h}$ with GNDF $\left(50 \mathrm{ng} \mathrm{mL} \mathrm{m}^{-1}\right)$ and lysed in PLB $1 \mathrm{X}$ (Passive Lysis Buffer) for the Dual-Luciferase Reporters assay (Promega). Firefly luciferase values were normalized to renilla luciferase values.

\section{Electromobility shift assay (EMSA) on mouse Apob -986bp region}

$10^{7}$ Neuro2a cells were rinsed in cold PBS- $\mathrm{Na}_{3} \mathrm{VO}_{4} 0.2 \mathrm{~mm}$, pelleted by centrifugation at $1600 \mathrm{~g}$ and lysed in $400 \mu \mathrm{L}$ of Buffer A $(10 \mathrm{~mm}$ HEPES $\mathrm{pH} 7.9,10 \mathrm{~mm} \mathrm{KCl}, 0.1 \mathrm{~mm}$ EDTA, $1 \mathrm{~mm}$ DTT, $1 \mathrm{~mm} \mathrm{Na} \mathrm{VO}_{4}$ and a protease inhibitors cocktail) for $15 \mathrm{~min}$ in ice. A quantity of $25 \mu \mathrm{L}$ of $10 \% \mathrm{NP}-40$ was added and the samples were centrifuged at $16000 \mathrm{~g} 30 \mathrm{~s}$. The nuclear pellets were resuspended in $50 \mu \mathrm{L}$ Buffer $\mathrm{C}(20 \mathrm{~mm}$ HEPES $\mathrm{pH} 7.9$, $0.4 \mathrm{~mm} \mathrm{KCl}, 0.1 \mathrm{~mm}$ EDTA, $0.1 \mathrm{~mm}$ EGTA, $1 \mathrm{~mm}$ DTT, $1 \mathrm{~mm}$ 
$\mathrm{Na}_{3} \mathrm{VO}_{4}$ and a protease inhibitors cocktail), incubated on ice for $45 \mathrm{~min}$, and centrifuged at $16000 \mathrm{~g} 10 \mathrm{~min}$ at $4{ }^{\circ} \mathrm{C}$. Supernatants were subjected to EMSA or stored at $-80^{\circ} \mathrm{C}$ until use. EMSA shift was performed with the LightShift Chemiluminescent EMSA Kit (Thermo Scientific Inc., Pittsburg, PA, USA). A quantity of $10 \mu \mathrm{g}$ of nuclear extracts, $20 \mathrm{fmol}$ of $5^{\prime}$ biotin-ATGCATTTCTATGCGTGCATGCCCATGAA probe, $50 \mathrm{ng}$ of poly(dI), $2.5 \%$ glycerol, $0.05 \% \mathrm{NP}-40$ and $5 \mathrm{~mm} \mathrm{MgCl}_{2}$, were incubated at room temperature for $20 \min$ in $20 \mu \mathrm{L}$. For the supershift assay, nuclear extracts were pre-incubated with anti-p53 mouse monoclonal antibody $(0.6 \mu \mathrm{g}$; Santa Cruz) on ice for $20 \mathrm{~min}$ prior to probe addition. For competition assay, 100 -fold excess unlabeled doublestranded oligonucleotides were incubated. Bound complexes were separated on $4 \%$ polyacrylamide gels, blotted onto nylon membrane and visualized according to the LightShift Chemiluminescent EMSA Kit.
A

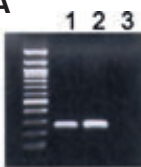

Ret

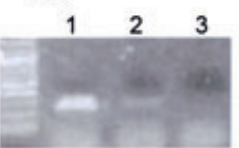

Ednrb

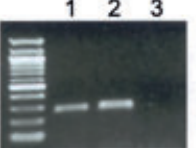

Gfra1

195 bp
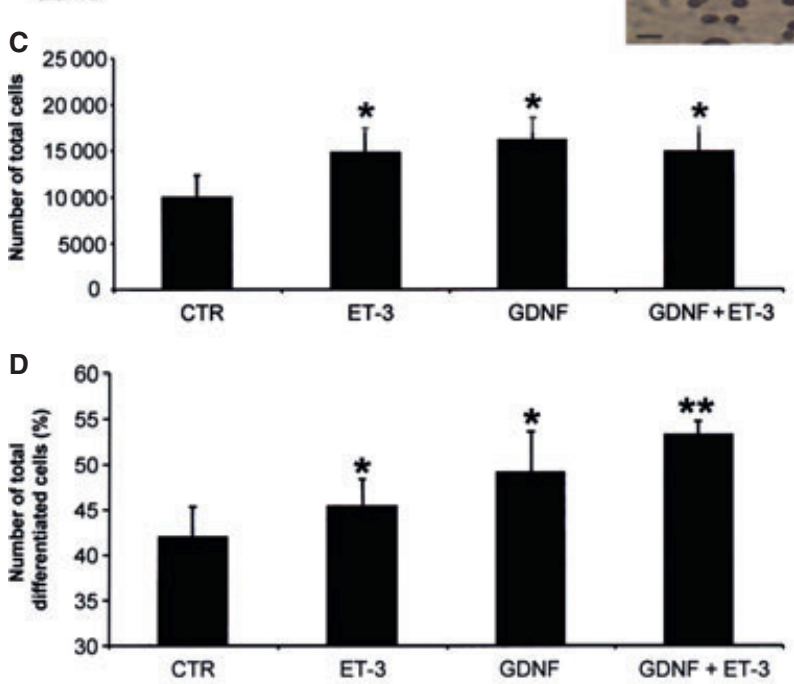

E

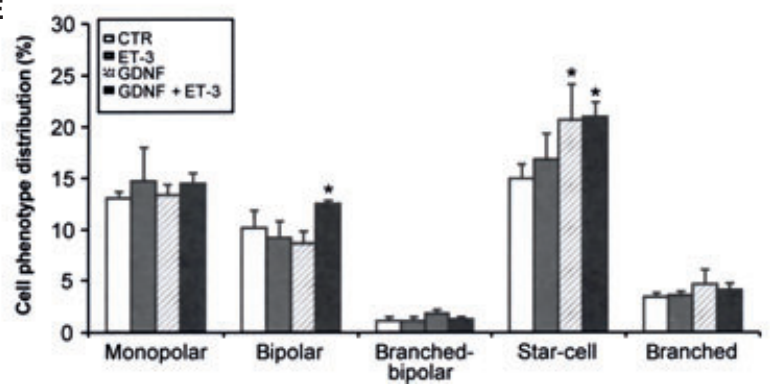

Figure 1 Effect of GDNF and ET-3 treatment on Neuro2a cell number and differentiation. (A) Expression of Ret, Gfra1, and Ednrb in mouse brain (1) and Neuro2a cells (2); (3) negative PCR controls. (B) Ret protein expression in Neuro2a cells. Immunolabeling with anti-Ret primary antibody of SK-N-MC that do not express Ret (1) and Neuro2a cell lines (3). (2, 4) Negative controls of NK-N-MC and Neuro2a cells, respectively. (C) Neuro2a cell numbers in cultures plated at moderate-high cell-plating densities at the end of a 4 days treatment with GDNF (10 ng mL ${ }^{-1}$ ) and ET-3 (300 nm separated and in combination. Data represent means \pm SE (D) Total differentiated Neuro2a cells in cultures plated at low cell-plating densities (2500 cells per well) at the end of a 4 days treatment with GDNF (10 ng mL ${ }^{-1}$ ) and ET-3 (300 nmol L ${ }^{-1}$ ) separated and in combination. (E) Cellphenotype distribution in the analyzed cells. Data represent percentage of differentiated cells $( \pm$ SE) observed in samples of 500 cells per replica-well for treatment conditions. Cells were classified in one of the six different cell phenotypes: monopolar, bipolar, branched-bipolar, star, and branched cells. 


\section{Statistical analysis}

Statistical analysis was performed using Statgraphics Plus 5.1. software (Statistical Graphics Corp., Warrenton, VA, USA). The Student's $t$-test was used to compare two groups, ANOva and chisquare test were used for multiple comparisons. A value of $P<0.05$ was considered significant. Data are expressed as means \pm SE.

\section{RESULTS}

ET-3/Ednrb signalling modulates GDNF/Retrelated cell growth and differentiation and $A p o b$ expression

We tested the existence of a crosstalk between Ednrb and Ret in Neuro2a cells and established whether or not these molecular pathways modulated Apob expression. The expression of Ret, Gdnfra, and Ednrb in Neuro2a cells has been verified by RT-PCR, whereas Ret protein has been detected using immunocytochemistry (Fig. 1A and B).

At a density of 5000 cells per well, the administration of GDNF exerted a more potent stimulatory effect on Neuro2a cell proliferation compared to ET-3 (ANOVA, $P<0.05$; chi-squared test, $P<0.0001)$. As expected, the concomitant administration of both ligands resulted in a lower Neuro2a cell proliferation due to molecular interference of the two pathways (Fig. 1C). At a density of 2500 cells per well, Neuro2a cells treated with GDNF and ET-3 for 4 days showed the most evident differentiation effect. Differentiated Neuro2a cells were classified into six different phenotypes (Figure S1). Differentiated cells were observed as a result of Neuro2a treatment with either GDNF or ET-3 (chisquared test, $P=8.04 \times \mathrm{e}^{-05}$ and $P=0.04$, respectively); the combination of the two ligands induced a stronger effect on the differentiation process (ANOva, $P<0.00001$; chi-squared test, $P=4.6 \times \mathrm{e}^{-10}$ ) (Fig. 1D). Cell-phenotype analysis indicated that after the combined GDNF and ET-3 treatment, cells were strongly induced to differentiate in two main phenotypes (i.e. bipolar and star-like) (chi-squared test, $P=0.034$ and $P=9.2 \times \mathrm{e}^{-06}$, respectively). GDNF alone induced cells to differentiate to the star-like phenotype (chi-squared test, $P=2.33 \times \mathrm{e}^{-05}$ ) (Fig. $1 \mathrm{E}$ ).

To determine whether or not $A p o b$ is modulated by GDNF/ET-3, we treated cells with the two ligands separately and in combination as reported in Fig. 2. We measured Apob mRNA levels through the qRT-PCR and data were normalized using the $\beta$-actin gene as endogenous control. GDNF induced an early strong increase in $A p o b$ expression after $30 \mathrm{~min}$ of stimulation (Fig. 2A), whereas, compared to GDNF, the treatment with ET-3 alone gave a less prominent Apob increment (Fig. 2B). The combination of GDNF and ET-3 led to an increase, although delayed in time, in Apob expression (Fig. 2C).

\section{Apob expression is activated by GDNF/RET signalling pathway}

To confirm that the increase in Apob expression was an exclusive effect of Ret activation, we silenced Ret by transient transfection with a Ret-specific short hairpin RNA. Transfected Neuro2a cells were treated with GDNF (50 $\left.\mathrm{ng} \mathrm{mL}^{-1}\right)$ at different times $(30 \mathrm{~min}$, $1 \mathrm{~h}$, and $3 \mathrm{~h}$ ), and protein and RNA were extracted. Neuro2a cells silenced for Ret did not show any Apob increase, confirming that the GDNF/Ret pathway activated $A p o b$ in a specific manner (Fig. 3A, upper panel). Western blotting confirmed the absence of Ret in the silenced cells (Fig. 3A, lower panel). Transfections with the empty or scramble vector did not abolish Ret-induced $A p o b$ increase (data not shown).

The inhibition of the GDNF/Ret-dependent MAPK P38 kinase with SB202190 completely abolished Apob
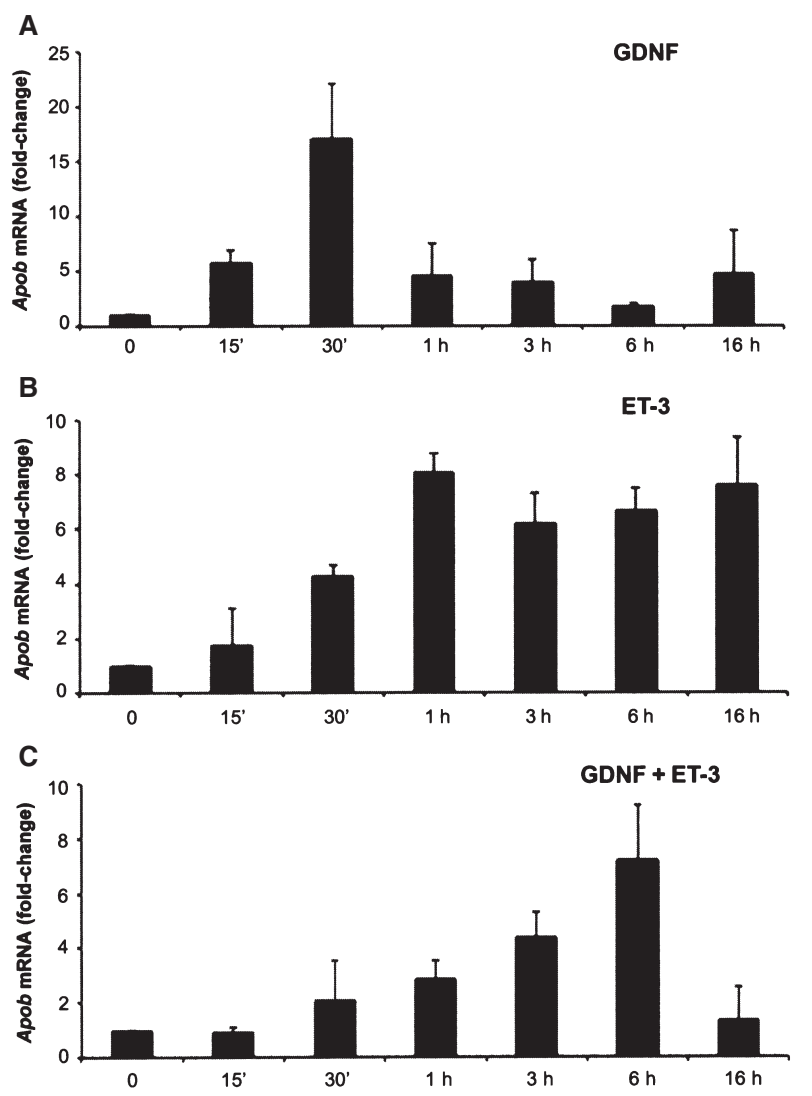

Figure 2 Effect of GDNF and ET-3 treatment on Apob expression. qRT-PCR of $A p o b$ in Neuro2a upon stimulation with GDNF (A), ET-3 (B) and GDNF and ET-3 in combination (C). Data represent the mean $( \pm \mathrm{SE})$ from three independent experiments. 
A

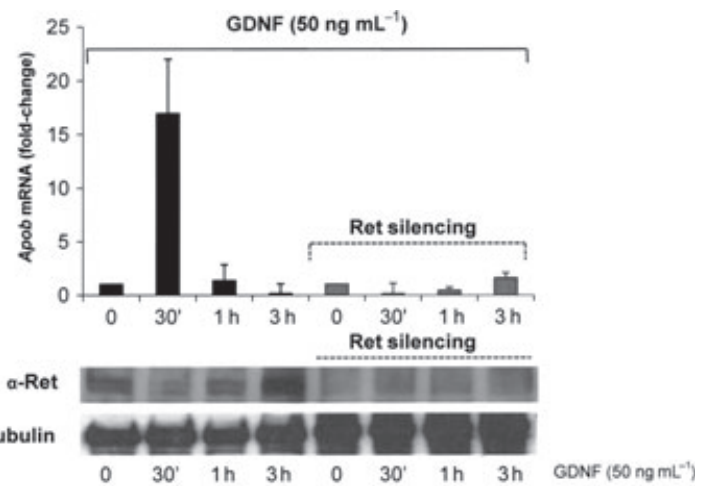

B
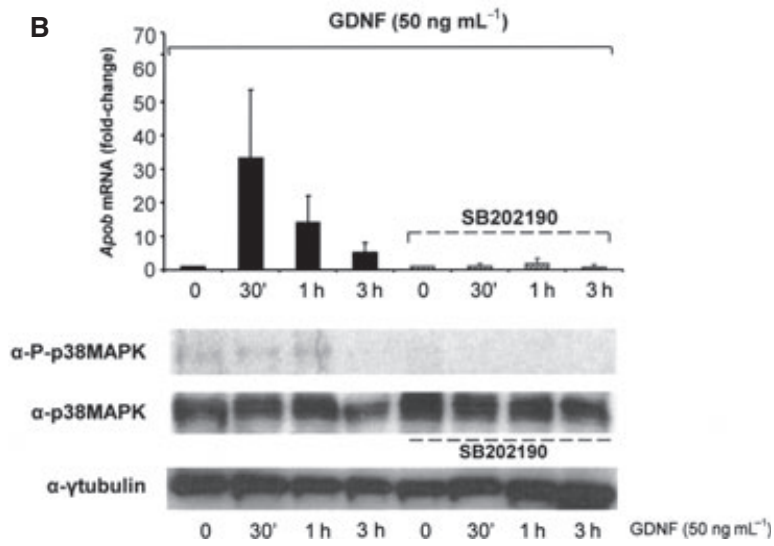

Figure 3 Specific activation of $A p o b$ transcription by RET/GDNF via MAPK P38 kinase. (A) Ret silencing abolished $A p o b$ increased expression in Neuro2a. Upper panel: qRT-PCR for Apob expression in GDNF-treated Neuro2a in GDNF-treated Neuro2a with Ret-silenced (dashed lines). The data represent the mean (error bars) from three independent experiments. Apob mRNA levels are normalized to Actb. Lower panel: Western blot analysis for Ret protein in serum starved GDNF-treated Neuro2a (first four lanes) and in serum-starved Neuro2a silenced for Ret and treated with GDNF (last 4 lanes). (B) Activation of Apob transcription by RET/GDNF via MAPK P38 kinase. Upper panel: qRT-PCR for Apob expression in GDNF-treated Neuro2a in absence and presence of SB202190 (dashed lines). Lower panel: Western blot analysis of GDNF-treated Neuro2a in absence or presence (last four lanes) of SB202190.

upregulation (Fig. 3B), whereas Akt and Erk kinase inhibitors did not modify its expression (Figure S2). Therefore, MAPK P38 kinase activation by Ret is responsible for the $A p o b$ increase.

\section{Apob silencing reduces $\mathrm{HuD}$ expression}

These experiments were aimed to understand whether or not Apob activation contributes to ENS development. In this line, we assessed HuD protein changes as an index of neuronal differentiation ${ }^{23}$ following GDNF treatment of Neuro2a cells transiently silenced for Apob. As shown in Fig. 4A and B, in GDNF-treated Neuro2a cells transfected with the scramble vector (control) the Apob-increased expression was associated with HuD protein increase, an effect prevented by Apob silencing.

\section{Expression analysis of Apob in HSCR mouse models}

To establish whether or not GDNF/Ret-induced $A p o b$ expression played a role on HSCR pathogenesis, we evaluated $A p o b$ expression in a mouse knock-in model for the Ret ${ }^{C 620 R}$ mutation. Ret ${ }^{C 620 R / C 620 R}$ mice show severe agangliogenesis, kidney agenesis, and die after birth, whereas the heterozygous mice show a normal organ development. ${ }^{19}$ We tested the expression of Apob in wild-type, heterozygous, and homozygous mouse embryos at day 16 via qRT-PCR (Fig. 5). Compared to wild-type, $A p o b$ expression was lower in homozygous mice (Student's $t$-test, $P=0.0372$ ), whereas there was a striking increase in expression in heterozygous mice (Student's $t$-test, $P=0.0052$ ) as a result of a functioning Ret. $^{19}$

\section{APOB immunolabeling in human colonic tissues}

Apolipoprotein B immunoreactivity was identified in cells (with morphological features of immunocytes) of the lamina propria of the adult normal human colon. In contrast, no APOB immunolabeling was detected in enteric neuronal cell bodies or nerve fibers, epithelial cells, or other cell targets of human colon tissue sections (Fig. 6).

\section{Identification of GDNF/Ret-dependent cis regulatory regions in mouse $A p o b$ promoter}

To determine the GNDF/Ret-dependent regulatory elements in the mouse $A p o b$ genomic region, we first predicted the promoter region in silico using two different softwares: Gene2promoter (http://www.genomatix.de) and PromoterScan. Both programs indicated the region between bp 4983663-4984504 of contig NT_039548 from mouse chromosome 12 as the predicted promoter. We cloned the region upstream of the mouse $A p o b$ gene, including the predicted $A p o b$ promoter, from genomic DNA and inserted it into the pGL3 vector containing the firefly luciferase gene as reporter. Constructs with progressive deletions of the cloned region were obtained by sequential digestion, and the plasmids were characterized by sequencing (Fig. 7A).

Each construct was co-transfected into Neuro2a cells with the control vector containing the renilla luciferase gene under the control of the SV40 promoter. One day after transfection, cells were serum starved for $16 \mathrm{~h}$ 
A

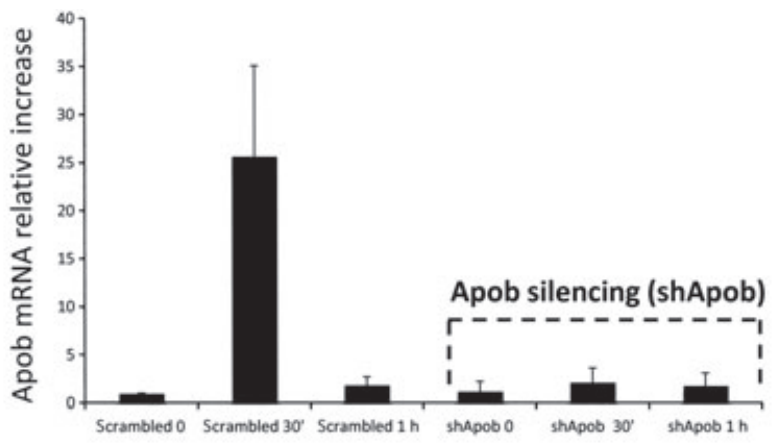

B Scrambled

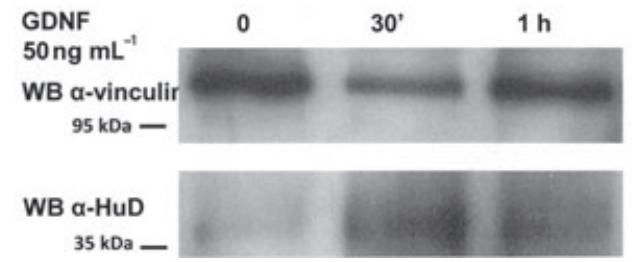

Apob silencing (shApob)

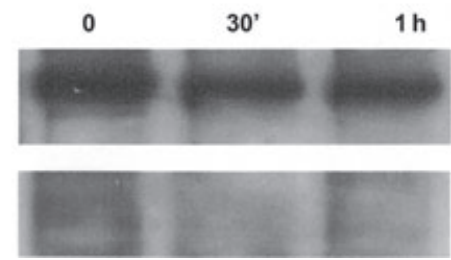

Figure 4 Apob silencing reduces HuD expression in GDNF-treated Neuro2a cells. (A) Relative Apob expression measured via real-time qRT-PCR in cells transfected with the scramble vector or with the shRNA vector for mouse Apob silencing (shApo) and treated with GDNF at different times. (B) HuD protein levels in Neuro2a cells transfected with scramble vector or with the shRNA vector for mouse Apob silencing (shApo) and treated with GDNF at different times (lower panel). Upper panel: Western blot for vinculin.

and GNDF stimulation was performed before cell lysis for the luciferase assay. No luciferase activity was found in absence of GDNF (Figure S3). All data were compared to the activity of the 900-bp construct, as this was the minimal promoter region defined by Gene2promoter and PromoterScan. The 1000-bp construct showed a significantly reduced activity compared to the 900-bp construct $(P=0.00020$, Student's $t$-test), suggesting that the $100 \mathrm{bp}$ extra-region contains repressor elements of Apob expression (Fig. 7B). to identify the cis regulatory elements responsible for Apob GDNF/Ret-dependent transcription regulation, we performed an in silico analysis of this region using MatInspector (http://www.genomatix.de). One site

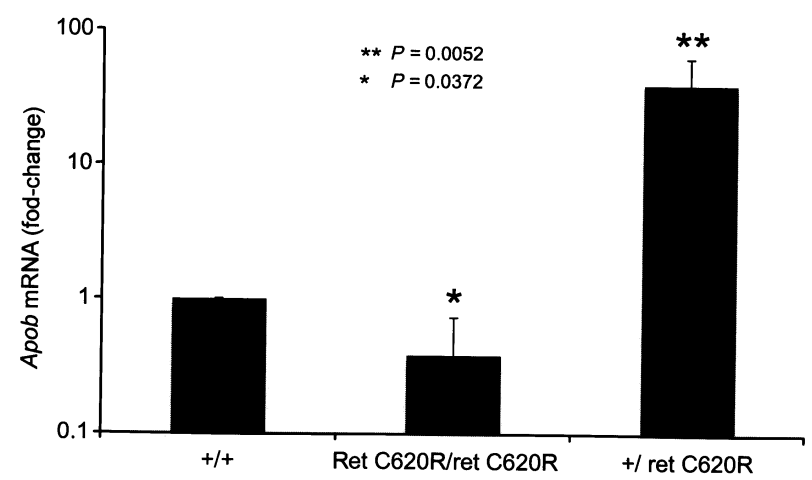

Figure 5 Apob expression in mouse embryos heterozygous and homozygous for Ret C620R (embryonic day 16.5) via qRT-PCR analysis. Data are expressed as means \pm SE. with a high matrix score (thus indicating a real binding site for transcription factors) was identified and represented a binding site for p53 (-986 bp site, matrix score $=0.934)($ Fig. 7C). Site-directed mutagenesis of the 1000-bp construct generated a deletion of $2 \mathrm{bp}$ in the p53-binding element (Fig. 7D; upper panel). Activities of wild-type and mutated 1000-bp constructs were compared to the activity of the 900-bp construct via the luciferase assay. The mutated 1000-bp construct showed a comparable activity with the 900-bp construct $(P=0.9457)$, whereas the original 1000-bp construct activity was reduced $(P \leq 0.0001)$ (Fig. 7D; lower panel). These data indicate that the p53-binding element acts as a repressor of $A p o b$ expression.

We performed an EMSA to verify that p53 actually binds to the region. As shown in Fig. 7E, we first verified that the binding of the Neuro2a nuclear extract to the biotin-labelled p53-putative binding site was specific, as it disappeared in the presence of the unlabelled probe (Fig. 7E, third lane). Next, we were able to detect a supershift when the nuclear extract was pre-incubated with an anti-p53 antibody (Fig. 7E), confirming the binding of p53 to the region.

\section{DISCUSSION}

RET and EDNRB are critical players during the development of the ENS, as mutations in genes encoding members of either RET or EDNRB pathways lead to a 

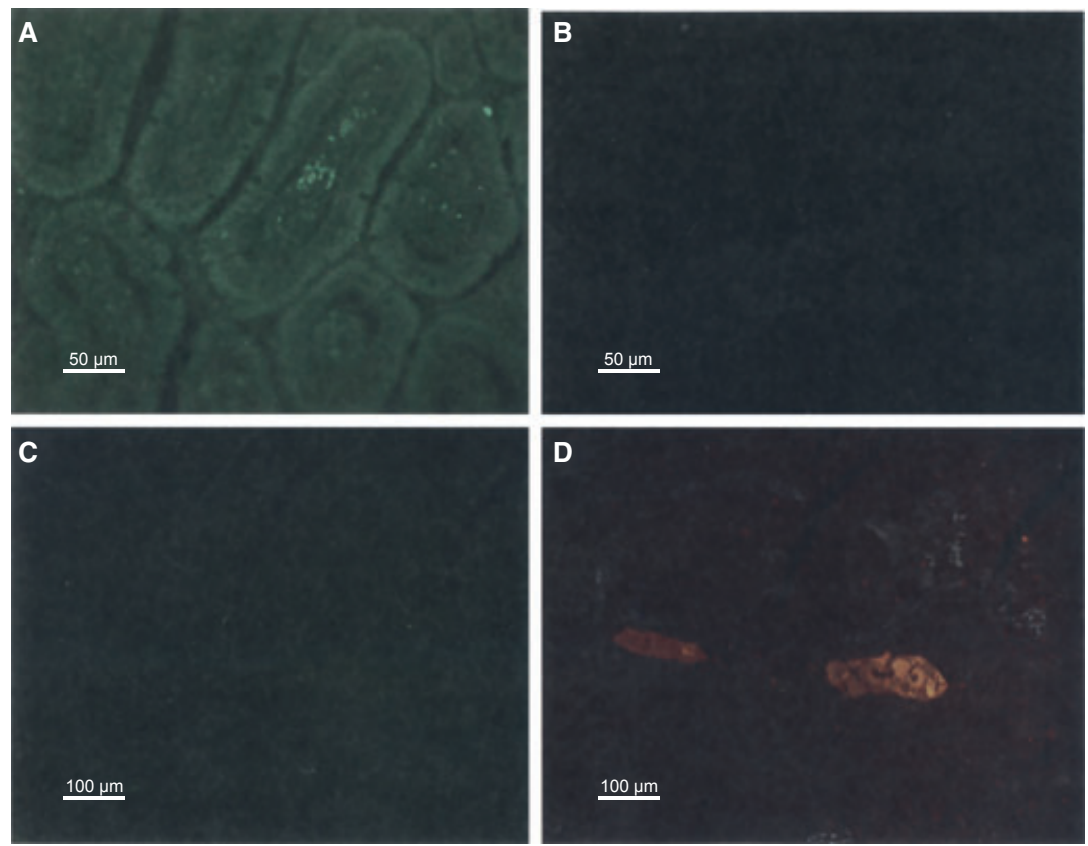

Figure 6 Representative examples showing APOB and NSE immunoreactivity in the adult human control colon. (A) Illustrates an intense APOB labeling in cells of lamina propria with features of immunocytes. (B) Shows the lack of APOB immunoreactivity as a control obtained by preincubating the primary antibody $(1: 100)$ with APOB peptide $\left(10^{-5} \mathrm{~m}\right)$. (C) Shows the lack of APOB immunoreactivity in the myenteric plexus which was clearly identified by NSE labeling (D). Mouse anti-goat and anti-rabbit secondary antibodies conjugated either with fluorescein isothiocyanate (FITC) or tetramethylrhodamine-isothiocyanate (TRITC) were used at $1: 300$ dilution each. Calibration bars: 50 and $100 \mu \mathrm{m}$, respectively.

HSCR phenotype. Although several studies have already identified genes that are modulated at the end of each signalling cascade and novel downstream genes activated by GDNF/RET have been identified, ${ }^{24,25}$ genes modulated by the crosstalk between these two pathways are still matter of investigation.

We have previously identified a vitellogenin (vit-3), corresponding to human $\mathrm{APOB}$, as a differentially expressed gene in C. elegans strains null for the worm homologue of mammalian ECE1. ${ }^{12,26}$ Several studies have shown the role of APOB in lipid transport and chylomicron production in the gut. ${ }^{13}$ Nevertheless, $A P O B$ mutations have been found in hypobetalipoproteinemia and defective apolipoprotein $\mathrm{B}-100^{14,15}$ and APOB levels have been mainly associated to the risk of chronic heart disease $\mathrm{e}^{27}$ and age-related macular degeneration. ${ }^{28}$ However, a possible correlation between $\mathrm{APOB}$ and neuronal function/development arises from studies in Apob homozygous null mice, which displayed neural tube defects and died in utero. ${ }^{16}$ Thus, the present study was undertaken to investigate whether or not Apob expression has a role in the proliferation/differentiation effects induced by Ret and Ednrb signalling pathways in the Neuro2a cell line, an in vitro model for the study of neuronal development and differentiation. ${ }^{17,18}$ We have demonstrated that
GDNF was a stronger inducer of cell proliferation compared to ET-3. When combined together, ET-3 partially inhibited the proliferative effect of GDNF, in accordance with previous results in enteric neural crest-derived cells committed to neuronal lineage. ${ }^{10}$ In our cell model, i.e. the Neuro2a cell line, the combination of GDNF and ET-3 induced cells to differentiate into bipolar and star-like phenotypes. Furthermore, GDNF promoted an early increase in Apob expression, which was delayed in combination with ET-3. These data paralleled the effect of GDNF/ET-3 on cell proliferation/differentiation described above, suggesting that the GDNF-mediated early Apob increase may be related to the proliferative effect on neural-crestderived cells.

We demonstrated that Apob-increased expression was a specific Ret-induced effect due to GDNF stimulation, because Ret silencing abolished Apob upregulation. This increase was dependent on the MAPK P38 kinase pathway, as MAPK P38 inhibition blocks Ret-induced $A p o b$ activation. MAPK P38 kinase plays a key role in the balance between cell proliferation and cell death induced by different stimuli. ${ }^{29}$ To gain further information on the downstream signals activated by Ret via MAPK P38, we evaluated the potential regulatory factors that mediated $A p o b$ increase. 
A

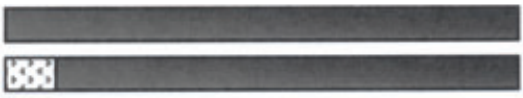

1000 bp Apob_prom $4983551-4984530$

900 bp Apob_prom 4983663-4984504
NT_039548 (mouse chr 12)

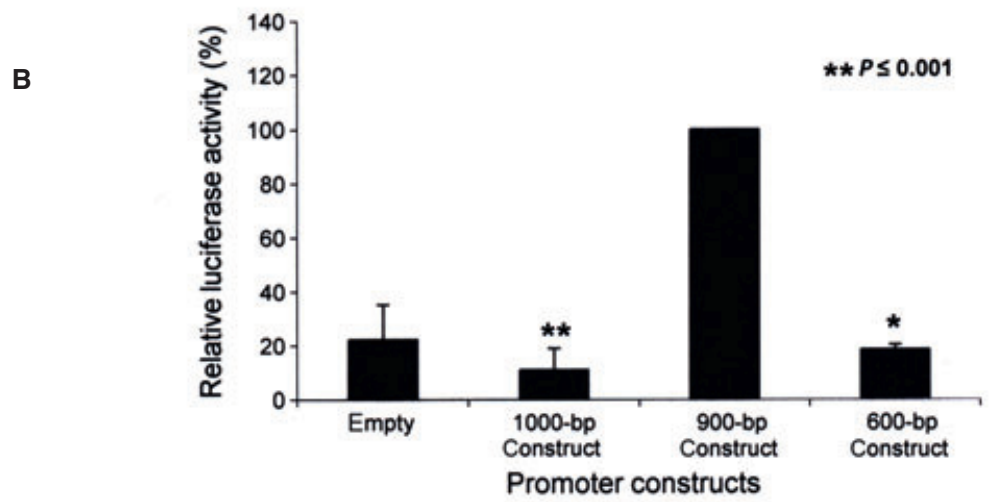

C

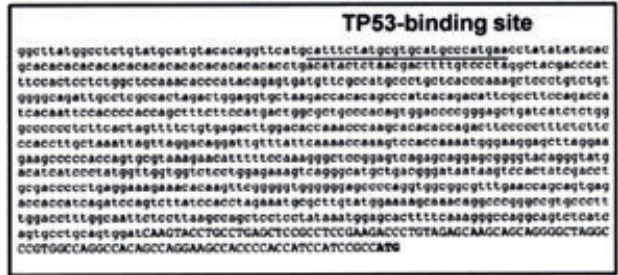

E

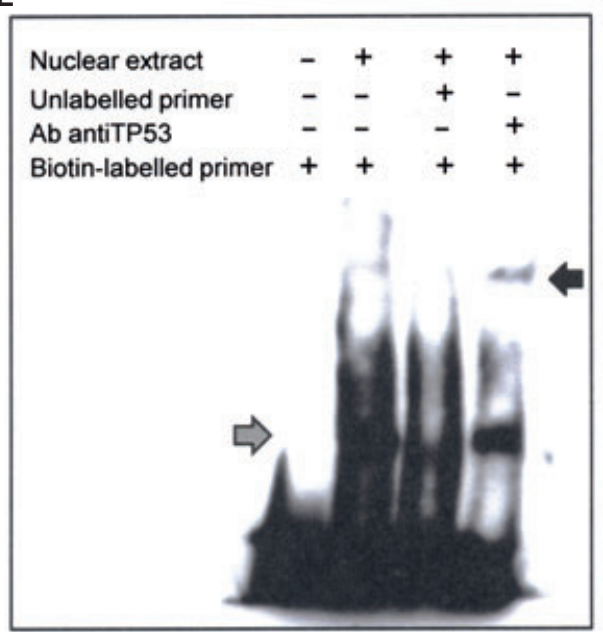

Figure 7 Identification of cis regulatory regions in mouse Apob promoter. (A) Map of the different constructs inserted in the pGL3 basic vector. (B) Results of the luciferase activity for the different constructs, compared to the predicted promoter (900-bp construct). Stars indicate the statistically significant different activities compared to the 900-bp construct. (C) Identification of p53 binding site in mouse $A p o B$ promoter. (D) Luciferase assay of the wild-type and mutant promoter regions (see text for details). (E) EMSA assay showing the binding of p53 (fourth lane, black arrow); gray arrow, complex forming in presence of Neuro2a nuclear extracts.

Although the downstream factors involved in the final regulation of $A p o b$ expression are still largely not known, we have proven that p53 is a key regulatory molecule for $A p o b$ expression. Luciferase and EMSA

\section{Wild type site Mutated site}

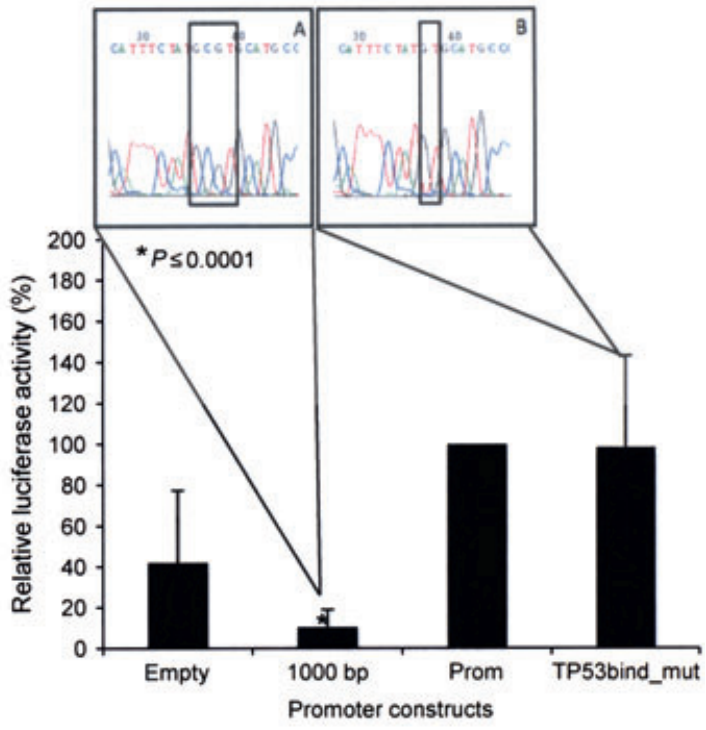

assays have shown that $\mathrm{p} 53$ acts as a repressor directly binding to the mouse promoter region of $A p o b$. Because MAPK P38 kinase induces p53 phosphorylation, ${ }^{30}$ it is likely that the GDNF/Ret pathway acti- 
vates the MAPK P38, which releases p53 from the promoter region thereby inducing Apob transcription. In support of this possibility recent data have indicated that p53 counteracts Ret activity during kidney development. ${ }^{31}$

Furthermore, we investigated whether or not $A p o b$ activation was related to enteric neuronal development by assessing $\mathrm{HuD}$ expression in GDNF-treated Neuro2a. $\mathrm{Hu}$ proteins are RNA-binding proteins (also referred to as $\mathrm{HuD}, \mathrm{HuC}, \mathrm{HuR}$, and $\mathrm{Hel}-\mathrm{N} 1$ ) that share sequence homology with the embryonic-lethal abnormal vision RNA-binding protein of Drosophila. In particular, $\mathrm{HuD}$ is exclusively expressed in neurons, where it exerts a crucial role in neuronal development and survival. ${ }^{32,33}$ In the present experiments, we provide evidence that $\mathrm{HuD}$ protein levels are linked to Apob expression. GDNF treatment of Neuro2a cells transfected with a control vector evoked Apob expression which was associated with $\mathrm{HuD}$ increase; in contrast, in $A p o b$ silenced Neuro2a cells GDNF did not increase HuD protein. Our data in Neuro2a cells provide support to the concept that $A p o b$ can contribute to enteric neuron differentiation and survival via $\mathrm{HuD}$ expression. In this line, early data by our group showed that anti-HuD antibodies incubated with primary culture of mature myenteric neurons evoked an apoptotic effect. ${ }^{34}$

In addition to the in vitro results, homozygous mice knock-in for the HSCR-associated Ret ${ }^{\mathrm{C} 620 \mathrm{R}}$ had a significant decrease of $A p o b$ expression compared to wild-type animals. In contrast, heterozygous mice, which have a normal differentiation and organization of the ENS, displayed a marked increase in Apob expression. The latter effect may be promoted by the wild-type Ret, to compensate for the loss of function of the mutated Ret isoform. In this line, recent data from our group showed that $A P O B$ mRNA and protein expression are increased in cells of the immune system and in serum of patients with chronic intestinal pseudo-obstruction compared to healthy controls (Bonora E and De Giorgio R, unpublished observation). The evidence that APOB expression can be detected in these cells is in agreement with data showing RET expression in the lymphoid tissues of the gut, where this receptor exerts an important regulatory function for Peyer's patch formation. ${ }^{35}$ Accordingly, in this study, APOB immunostaining was selectively detected in cells, most likely of the immune system, distributed throughout the lamina propria of the normal adult colon.

To our knowledge, this is the first report dealing with Ret-related $A p o b$ expression in both in vitro (Neuro2a cells) and in vivo (Ret C620R knock-in mouse) models. More broadly, it may be proposed that RET-dependent $A P O B$ expression exerts a major role in regulating different molecular pathways required for enteric neurogenesis and other tissue development. Likewise RET, also APOB may influence the immunoregulatory function in the digestive system, although this exciting aspect clearly deserves further investigation. The data herein presented provide a conceptual framework to evaluate APOB in patients with severe functional gastrointestinal disorders characterized by an impaired motility and transit.

\section{ACKNOWLEDGMENTS}

We thank Dr Kerry J. Rhoden for critical reading and helpful suggestions and Ms Silvia Cerboni for technical help.

\section{FUNDING}

This work was supported by FP6 EU grant LSHC-CT-2006-037530 'HERMIONE' and partially supported by FP7 EU grant 223692 'CHERISH' to G. R. This work was also partly supported by grants from the Italian Ministry of University and Research (COFIN Projects to R. De G.), Ricerca Fondamentale Orientata funds from the University of Bologna to R. De G., V. S. and (Centro Interdipartimentale di Ricerca sull'Alimentazione Umana, CIRAU) funds to R. De G. R. De G. is a recipient of grants from Fondazione Del Monte di Bologna e Ravenna, Bologna, Italy. C. E. was a recipient of University of Torino doctoral fellowship. L. M. P. was supported by the Sardininian 'Master and Back' project Fellowship.

\section{DISCLOSURE}

The authors declare no competing interests.

\section{AUTHOR CONTRIBUTION}

$\mathrm{CE}$ performed the research, analyzed and interpreted the data and wrote the paper; FB, LMP, MR performed the research; AP, IS, AG, MV analyzed and interpreted the data; MS, GR, EB designed the experimental plan and wrote the paper; RDeG and VS revised the whole manuscript and contributed to experimental analysis.

\section{REFERENCES}

1 Gershon MD. Developmental determinants of the independence and complexity of the enteric nervous system. Trends Neurosci 2010; 33: 446-56.
2 Panza E, Knowles CH, Graziano C et al. Genetics of human enteric neuropathies. Prog Neurobiol 2012; 96: $176-89$. 
3 Amiel I, Lyonnet S. Hirschsprung disease, associated syndromes, and genetics: a review. J Med Genet 2001; 38: 729-39.

4 Arighi E, Borrello MG, Sariola H. RET tyrosine kinase signaling in development and cancer. Cytokine Growth Factor Rev 2005; 16: 441-67.

5 Emison ES, McCallion AS, Kashuk CS et al. A common sex-dependent mutation in a RET enhancer underlies Hirschsprung disease risk. Nature 2005; 434: 857-63.

6 Tahira T, Ishizaka Y, Itoh F, Sugimura $T$, Nagao $M$. Characterization of ret proto-oncogene mRNAs encoding two isoforms of the protein product in a human neuroblastoma cell line. Oncogene 1990; 5: 97-102.

7 Arai H, Hori S, Aramori I, Ohkubo H, Nakanishi S. Cloning and expression of a cDNA encoding an endothelin receptor. Nature 1990; 348: 730-2.

8 Carrasquillo MM, McCallion AS, Puffenberger EG, Kashuk CS, Nouri $\mathrm{N}$, Chakravarti A. Genome-wide association study and mouse model identify interaction between RET and EDNRB pathways in Hirschsprung disease. Nat Genet 2002; 32: 237-44.

9 McCallion AS, Stames E, Conlon RA, Chakravarti A. Phenotype variation in two-locus mouse models of Hirschsprung disease: tissue-specific interaction between Ret and Ednrb. Proc Natl Acad Sci U S A 2003; 100: 1826-31.

10 Barlow A, de GraaffE, Pachnis V. Enteric nervous system progenitors are coordinately controlled by the $\mathrm{G}$ protein-coupled receptor EDNRB and the receptor tyrosine kinase RET. Neuron 2003; 40: 905-16.

11 Kruger GM, Mosher JT, Tsai YH et al. Temporally distinct requirements for endothelin receptor B in the generation and migration of gut neural crest stem cells. Neuron 2003; 40: 917-29.

12 Spanier B, Stürzenbaum SR, HoldenDye LM, Baumeister R. Caenorhabditis elegans neprilysin NEP-1: an effector of locomotion and pharyngeal pumping. I Mol Biol 2005; 352: 429-37.

13 Rutledge AC, Su Q, Adeli K. Apolipoprotein $\mathrm{B} 100$ biogenesis: a complex array of intracellular mechanisms regulating folding, stability, and lipoprotein assembly. Biochem Cell Biol 2010; 88: 251-67.
14 Linton MF, Farese RV Jr, Young SG. Familial hypobetalipoproteinemia. I Lipid Res 1993; 34: 521-41.

15 Soria LF, Ludwig EH, Clarke HRG, Vega GL, Grundy SM, McCarthy BJ. Association between a specific apolipoprotein $\mathrm{B}$ mutation and familial defective apolipoprotein B-100. Proc Natl Acad Sci U S A 1989; 86: 587-91.

16 Huang LS, Voyiaziakis E, Markenson DF, Sokol KA, Hayek T, Breslow JL. apo $\mathrm{B}$ gene knockout in mice results in embryonic lethality in homozygotes and neural tube defects, male infertility, and reduced HDL cholesterol ester and apo A-I transport rates in heterozygotes. I Clin Invest 1995; 96: 2152-61.

17 Lin $\mathrm{CH}$, Hsieh $\mathrm{M}$, Fan SS. The promotion of neurite formation in Neuro2A cells by mouse Mob2 protein. FEBS Lett 2011; 585: 523-30.

18 Sanchez-Ferras O, Coutaud B, Djavanbakht SamaniT, Tremblay I, Souchkova O, Pilon N. Caudalrelated homeobox $(\mathrm{Cdx})$ proteindependent integration of canonical Wnt signaling on paired-box 3 ( $\operatorname{Pax} 3)$ neural crest enhancer. I Biol Chem 2012; 287: 16623-35.

19 Yin L, Puliti A, Bonora E et al. C620R mutation of the murine ret protooncogene: loss of function effect in homozygotes and possible gain of function effect in heterozygotes. Int Cancer 2007; 121: 292-300.

20 Mograbi B, Bocciardi R, Bourget I et al. Glial cell line-derived neurotrophic factor-stimulated phosphatidylinositol 3-kinase and Akt activities exert opposing effects on the ERK pathway: importance for the rescue of neuroectodermic cells. Biol Chem 2001; 276: 45307-19.

21 Bunone G, Borrello MG, Picetti R et al. Induction of RET proto-oncogene expression in neuroblastoma cells precedes neuronal differentiation and is not mediated by protein synthesis. Exp Cell Res 1995; 217: 92-9.

22 De GiorgioR, Volta U, Stanghellini V et al. Neurogenic chronic intestinal pseudo-obstruction: antineuronal antibody-mediated activation of autophagy via Fas. Gastroenterology 2008; 135: 601-9.

23 Liu MT, Kuan YH, Wang J, Hen R, Gershon MD. 5-HT4 receptor-mediated neuroprotection and neurogenesis in the enteric nervous system of adult mice. I Neurosci 2009; 29: 9683-99.

24 Heanue TA, Pachnis V. Expression profiling the developing mammalian enteric nervous system identifies marker and candidate Hirschsprung disease genes. Proc Natl Acad Sci U S A 2006; 103: 6919-24.

25 Lu BC, Cebrian C, Chi X et al. Etv4 and Etv5 are required downstream of GDNF and Ret for kidney branching morphogenesis. Nat Genet 2009; 41: 1295-302.

26 Bonora E, Evangelisti C, Puliti A et al. Apolipoprotein $\mathrm{B}$ is a new target of the RET/GDNF signalling pathway -ESHG, 2011; A-863 (Abstract).

27 Cromwell WC, Barringer TA. Lowdensity lipoprotein and apolipoprotein B: clinical use in patients with coronary heart disease. Curr Cardiol Rep 2009; 11: 468-75.

28 Fujihara M, Bartels E, Nielsen LB, Handa JT. A human apoB100 transgenic mouse expresses human apoB100 in the RPE and develops features of early AMD. Exp Eye Res 2009; 88: 1115-23.

29 Feng Y, Wen J, Chang CC. p38 Mitogen-activated protein kinase and hematologic malignancies. Arch Pathol Lab Med 2009; 133: 850-6.

30 Brown L, Benchimol S. The involvement of MAPK signaling pathways in determining the cellular response to p53 activation: cell cycle arrest or apoptosis. I Biol Chem 2006; 281: 3832-40.

31 Hilliard S, Aboudehen K, Yao X, El-Dahr SS. Tight regulation of p53 activity by $\mathrm{Mdm} 2$ is required for ureteric bud growth and branching. Dev Biol 2011; 353: 354-66.

32 Okano HJ, Darnell RB. A hierarchy of $\mathrm{Hu}$ RNA binding proteins in developing and adult neurons. I Neurosci 1997; 17: 3024-37.

33 Wakamatsu Y, Weston JA. Sequential expression and role of $\mathrm{Hu}$ RNAbinding proteins during neurogenesis. Development 1997; 124: 3449-60.

34 De GiorgioR, Bovara M, Barbara G et al. Anti-HuD-induced neuronal apoptosis underlying paraneoplastic gut dysmotility. Gastroenterology 2003; 125: 70-9.

35 Veiga-Fernandes H, Coles MC, Foster $\mathrm{KE}$ et al. Tyrosine kinase receptor RET is a key regulator of Peyer's patch organogenesis. Nature 2007; 446: $547-51$. 


\section{SUPPORTING INFORMATION}

Additional Supporting Information may be found in the online version of this article:

Figure S1. Cell morphology classification. Cells were classified according to the presence and shape of neurites as follows: undifferentiated, round-shaped cells (A); with one neurite (monopolar) (B), with two opposite neurites (bipolar) (C); with two opposite and branched neurites (branched-bipolar) (D), with many neurites (star cells) (E); with one prevalent branched neurite (branched) (F).

Figure S2. (A) Western blot analysis of GDNF-treated Neuro2a in absence or presence (last 4 lanes) of Triciribine V, an Akt inhibitor. (B) qRT-PCR for Apob expression in GDNF-treated Neuro2a in presence of Triciribine V. (C) Western blot analysis of GDNF-treated Neuro2a in absence or presence (last 4 lanes) of PD98059, a specific ERK inhibitor. (F) qRT-PCR for Apob expression in GDNF-treated Neuro2a in presence of PD98059.

Figure S3. Luciferase activity assay for the different constructs, compared to the predicted promoter $(900 \mathrm{bp}$ construct), in absence of GDNF stimulation.

Please note: Wiley-Blackwell are not responsible for the content or functionality of any supporting materials supplied by the authors. Any queries (other than missing material) should be directed to the corresponding author of the article. 